\title{
Dependence of size distribution for liquid cluster ions on retarding voltage
}

\section{AUTHOR(S):}

Takaoka, Gikan H.; Okada, Takeshi; Sugiyama, Kazumichi; Kawashita, Masakazu

\section{CITATION:}

Takaoka, Gikan H. ... [et al]. Dependence of size distribution for liquid cluster ions on retarding voltage. REVIEW OF SCIENTIFIC INSTRUMENTS 2008, 79(2(Part 2)): 02 B305.

\section{ISSUE DATE:}

2008-02

URL:

http://hdl.handle.net/2433/84586

\section{RIGHT:}

Copyright 2008 American Institute of Physics. This article may be downloaded for personal use only. Any other use requires prior permission of the author and the American Institute of Physics. 


\title{
Dependence of size distribution for liquid cluster ions on retarding voltage $\mathrm{e}^{\text {a) }}$
}

\author{
Gikan H. Takaoka, ${ }^{\text {b) }}$ Takeshi Okada, Kazumichi Sugiyama, and Masakazu Kawashita \\ Photonics and Electronics Science and Engineering Center, Kyoto University, Nishikyo, \\ Kyoto 615-8510, Japan
}

(Received 16 August 2007; presented 29 August 2007; accepted 12 September 2007; published online 18 January 2008)

\begin{abstract}
We have succeeded in producing liquid clusters such as ethanol and water clusters by an adiabatic expansion phenomenon, and the cluster size distribution was analyzed by using the time-of-flight method. For ethanol cluster ions, the size was distributed between a few hundreds and a few thousands, and the peak size was approximately 1200 molecules per cluster. For water cluster ions, the size distribution shifted to higher values, and the peak size was approximately 2500 molecules per cluster. Furthermore, the cluster size distribution was measured by changing the retarding voltage, and it was found that the minimum size increased with increase of the retarding voltage. In addition, the minimum size was larger than the critical size estimated by the theoretical calculation. (C) 2008 American Institute of Physics. [DOI: 10.1063/1.2801547]
\end{abstract}

\section{INTRODUCTION}

A cluster is an aggregate of atoms (or molecules) with the diameter of a few nanometers and constitutes a new phase of matter, with significantly different properties than solids, liquids, and gases. ${ }^{1,2}$ The investigations of the clusters open a new field of material science, which bridges the gap between individual atoms and bulk state matter. For the liquid clusters such as water and alcohol clusters, their physical and chemical properties, which are considered to change according to the cluster size, are also different from those of the bulk state. ${ }^{3-5}$ In addition, impact of the liquid cluster ions on solid surfaces has exhibited unique irradiation effects. ${ }^{6-8}$ For example, high density irradiation effect is predominant, which results in achieving high-rate sputtering of the solid surfaces. Another is extremely low energy irradiation effect, and the average energy of the molecule in the cluster ion is estimated to be the incident energy of the cluster ions divided by the cluster size. When the cluster ions with cluster size of 1000 molecules are irradiated on the surface at an acceleration voltage of $1 \mathrm{kV}$, the incident energy of the molecule in the cluster ion is $1 \mathrm{eV}$ per molecule. Therefore, the irradiation damage by the cluster ion beams is very low compared with that by Ar monomer ion beams.

The irradiation effects mentioned above are different depending on the incident energy and the cluster size. In order to clarify the size dependence of the impact process as well as the property of the cluster ion itself, it is of much importance to control the cluster size distribution. In this article, the size distribution of ethanol and water cluster ions is investigated by using both the time-of-flight (TOF) method and the retarding potential method, and the retarding voltage dependence for the size distribution is described. Furthermore,

\footnotetext{
a) Contributed paper, published as part of the Proceedings of the 12th International Conference on Ion Sources, Jeju, Korea, August 2007.

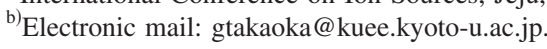

the minimum size measured for the ethanol cluster ions is discussed based on the experimental result and the theoretical estimation.

\section{EXPERIMENTAL PROCEDURE}

When vapors of liquid materials were ejected through a nozzle into a vacuum region, vaporized clusters were produced by an adiabatic expansion phenomenon. The liquid clusters produced entered an ionizer and were ionized by electron bombardment. Figure 1 shows the schematic diagram of the ionizer and the lens in the liquid cluster ion beam system. As shown in the figure, electrons are emitted from a hot filament of a tungsten wire and accelerated by a voltage applied between the filament and the cylindrical anode. The neutral clusters entering the anode are ionized by electron bombardment. The electron voltage for ionization $\left(V_{e}\right)$ was adjusted between 0 and $300 \mathrm{~V}$, and the electron current for ionization $\left(I_{e}\right)$ was adjusted between 0 and $250 \mathrm{~mA}$. The cluster ions are extracted by applying an acceleration voltage to the anode. The extracted cluster ions are size separated by a retarding potential method. The retarding voltage, which is determined as a voltage $\left(V_{r}\right)$ between the anode and the retarding electrode, can be adjusted by the voltage applied to the retarding electrode. In order to make the electric potential near the beam axis constant, the retarding electrode as well as the extraction electrode has a sheet of nickel mesh in the aperture. The size-separated cluster ion beams are focused by applying a lens voltage $\left(V_{l}\right)$ to the Einzel lens and are accelerated toward a Faraday cup. The acceleration voltage $\left(V_{a}\right)$ was adjusted between 0 and $10 \mathrm{kV}$. The retarding voltage $\left(V_{r}\right)$ was adjusted between 0 and $168 \mathrm{~V}$. The background pressure around the Faraday cup was $1 \times 10^{-7}$ Torr, which was attained using a turbomolecular pump.

In the cluster formation process by the adiabatic expan- 


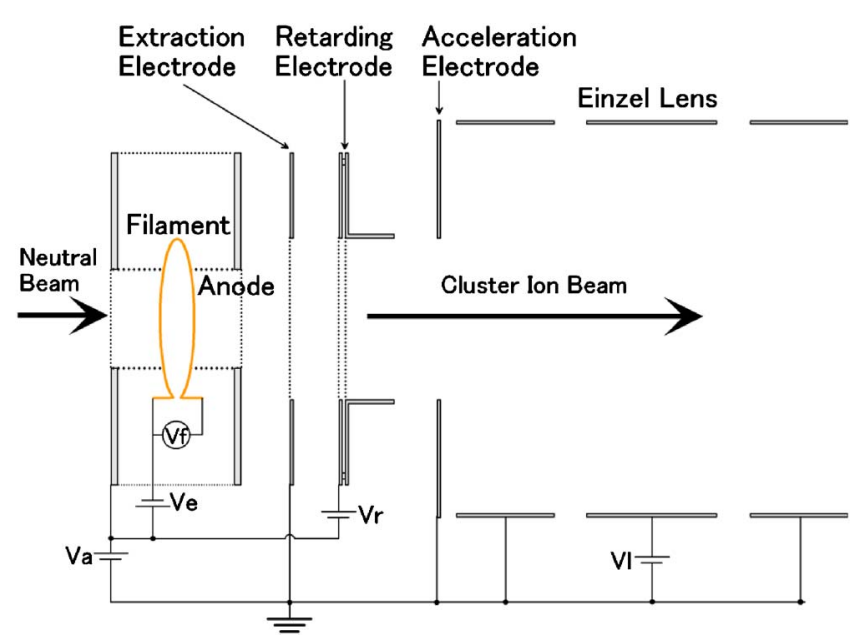

FIG. 1. (Color online) Schematic diagram of the liquid cluster ion beam apparatus.

sion phenomenon, the thermal random energy of the vapors is transferred to the drift velocity. The drift velocity of the cluster can be estimated from the kinetic energy of the cluster. The kinetic energy $E_{N}$ of the cluster with cluster size of $N$ is described as follows: ${ }^{\prime}$

$$
E_{N}=\frac{1}{2} N m v^{2}=\frac{\gamma}{\gamma-1} k T_{0} N,
$$

where $m$ is the mass of the source materials, $v$ is the drift velocity of the cluster, $\gamma$ is the specific heat ratio, $k$ is the Boltzmann constant, and $T_{0}$ is the temperature of the source materials. When the retarding voltage $\left(V_{r}\right)$ is applied to a retarding electrode, the cluster ions smaller than the critical size calculated from the equation $E_{N}=e V_{r}$ cannot pass through the retarding electrode. Here $e$ is the electric charge. Therefore, the minimum size of the cluster ions can be adjusted by changing the retarding voltage.

With regard to the cluster size distribution for the liquid cluster ions, it was measured by a TOF method. In the TOF measurement, it was assumed that the cluster ion had a single charge, and multiply charged cluster ions might be dissociated due to the Coulomb repulsion force. As already shown in Fig. 1, the cluster ions are extracted from the ionizer and pass through the retarding electrode. Thereafter, they are accelerated and enter a flight tube in the TOF instrument. During drift in the flight tube, they are deflected by a pulsed negative voltage. When the pulsed voltage is applied, the cluster ions drift toward a Faraday cup mounted at the end of the flight tube. The flight ions are detected by the Faraday cup. In the TOF measurement, the acceleration voltage for the cluster ions was at 6 and $9 \mathrm{kV}$, and the pulsed voltage was $2 \mathrm{kV}$. The duration time and the repetition rate of the pulsed voltage were $10 \mu \mathrm{s}$ and $100 \mathrm{~Hz}$, respectively. The drift distance was $0.51 \mathrm{~m}$. The cluster size was calculated based on the drift time ranging from microseconds to milliseconds, which was different depending on the mass.

\section{RESULTS AND DISCUSSION}

Figure 2 shows the cluster size distribution measured at different retarding voltages for (a) ethanol cluster ions and

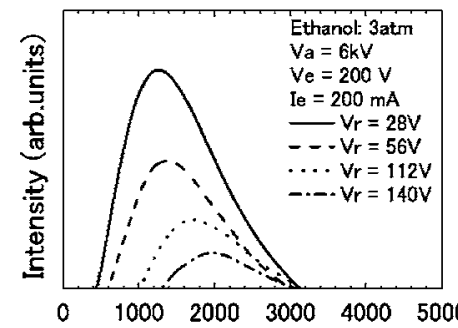

(a) Cluster Size (molecules)
FIG. 2. Cluster size distribution at different retarding voltages for (a) ethanol cluster ions and (b) water cluster ions.

(b) water cluster ions. The source temperature was $109^{\circ} \mathrm{C}$ for ethanol and $134{ }^{\circ} \mathrm{C}$ for water. The vapor pressure of ethanol and water was $3 \mathrm{~atm}$. The acceleration voltage was $6 \mathrm{kV}$. As shown in the figure, the peak size is approximately 1200 molecules for ethanol cluster ions and approximately 2500 molecules for water cluster ions. The specific heat ratio of water and ethanol is 1.33 and 1.13 , respectively, and the difference in the heat specific ratio influences the cluster formation process. Furthermore, the minimum size as well as the peak size of the cluster ions increases with increase of the retarding voltage, although the intensity decreases with increase of the retarding voltage. This indicates that the cluster ions with smaller size are removed from the ion beams by applying the retarding voltage.

The size distribution of the ethanol cluster ions was measured by changing the acceleration voltage and the retarding voltage. As shown in Fig. 3, the minimum size increases with increase of the retarding voltage. For acceleration voltages such as 6 and $9 \mathrm{kV}$, the minimum size measured is larger than the critical size calculated from Eq. (1). For example, the minimum size at a retarding voltage of $28 \mathrm{~V}$ is approximately 400 molecules, although the calculated size is 100 molecules. For the theoretical calculation, it was assumed that all the cluster ions were transferred in parallel to

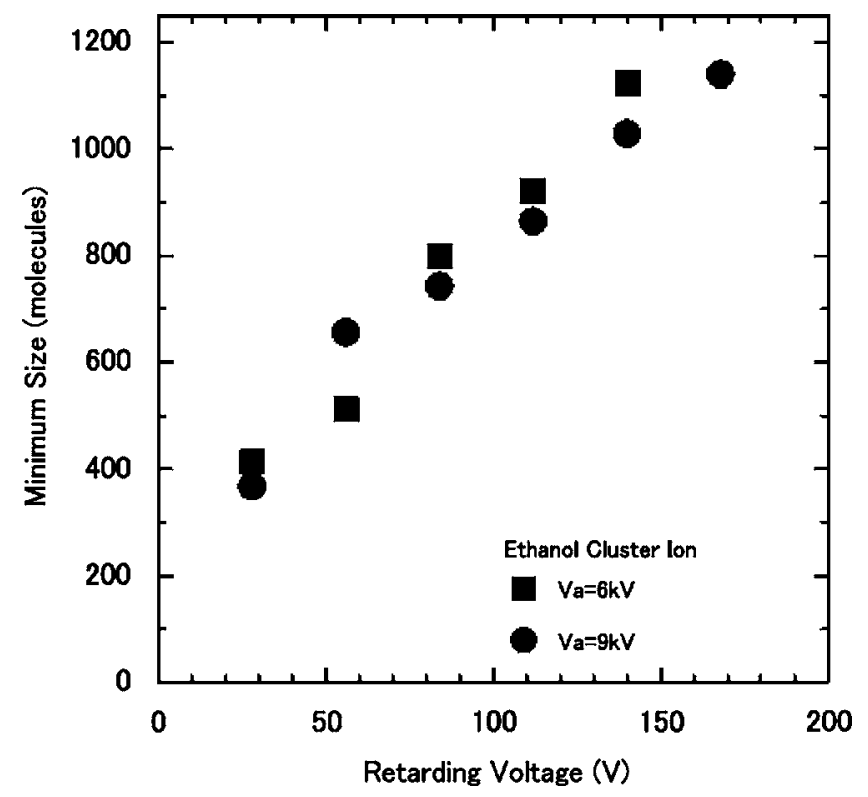

FIG. 3. Dependence of minimum size of ethanol cluster ions on retarding voltage. 


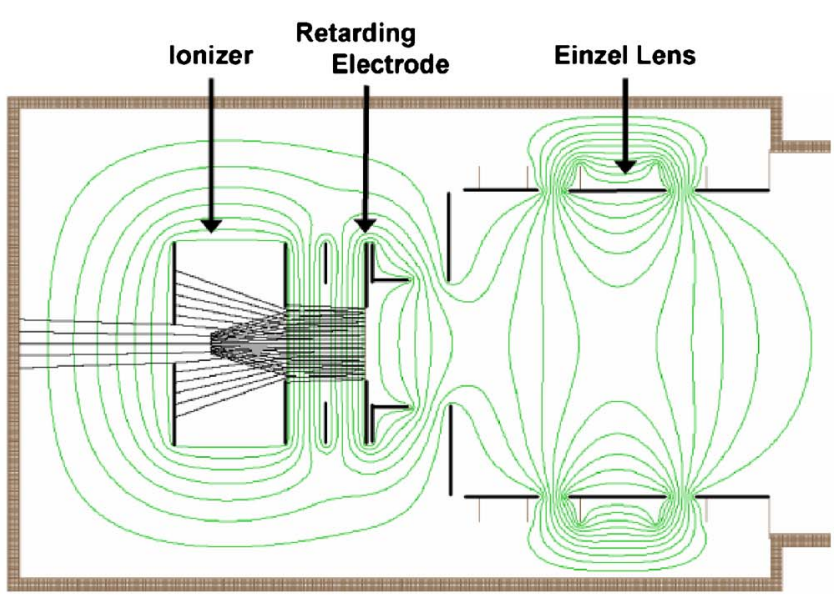

(a) Size:100molecules

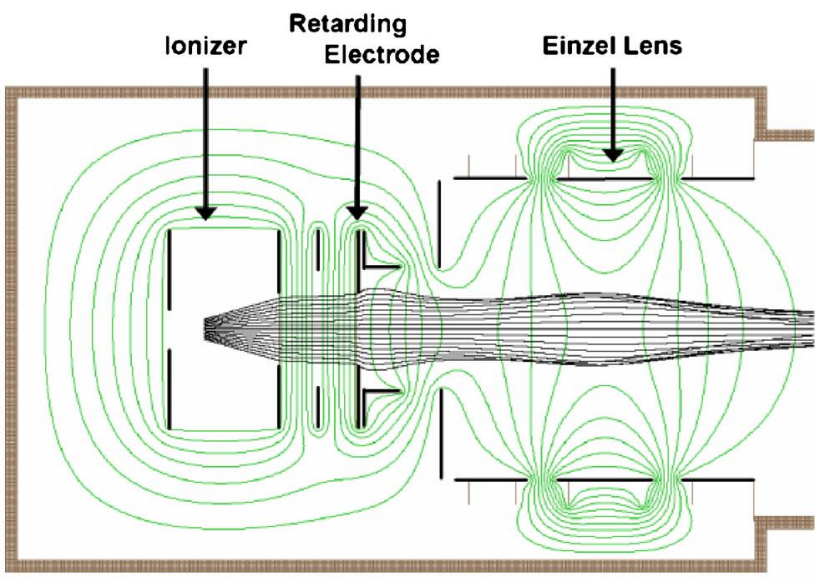

(b) Size:200molecules

FIG. 4. (Color online) Beam trajectories for the ethanol cluster ion beams with cluster sizes of (a) 100 molecules and (b) 200 molecules.

the beam axis toward the Faraday cup. If some cluster ions in the experimental setup might be divergent due to the space charge effect or the electric field deformation, even the cluster ions larger than the critical size would be reflected at the retarding electrode. However, the computer simulation showed that the possibility of the reflection was very small.

Figure 4 shows the beam trajectories simulated for the ethanol cluster ion beams with cluster sizes of (a) 100 molecules and (b) 200 molecules. In the figure, equipotential lines around the ionizer and the Einzel lens are also shown in thin lines, and the ion beams with a divergent angle of $20^{\circ}$ are ejected from the central part of the ionizer. The electron voltage for ionization was kept at $V_{e}=200 \mathrm{~V}$. The acceleration voltage and the retarding voltage were at $V_{a}=6 \mathrm{kV}$ and $V_{r}=28 \mathrm{~V}$, respectively. In addition, the lens voltage $\left(V_{l}\right)$ applied to the Einzel lens was $9 \mathrm{kV}$. As shown in the figure, ethanol cluster ions with a cluster size of 100 molecules are reflected at the retarding electrode, but the cluster ions with a cluster size of 200 molecules pass through it. Therefore, the measurement of the minimum size larger than the calculated size, which is shown in Fig. 3, is not due to the divergence of the cluster ion beams, and it might be due to another reason such as overestimation of the drift velocity of the cluster ions.

In summary, we produced liquid clusters such as ethanol and water clusters by an adiabatic expansion phenomenon, and the cluster size distribution was analyzed by using both the TOF method and the retarding potential method. For ethanol cluster ions, the size was distributed between a few hundreds and a few thousands, and the peak size was approximately 1200 molecules per cluster. For water cluster ions, the size distribution shifted to higher values, and the peak size was approximately 2500 molecules per cluster. With regard to the cluster size distribution measured by changing the retarding voltage, it was found that the minimum size increased with increase of the retarding voltage. The minimum size was approximately 400 molecules per cluster at $V_{r}=28 \mathrm{~V}$ for the ethanol cluster ions, and it was approximately 600 molecules per cluster at $V_{r}=14 \mathrm{~V}$ for the water cluster ions. These minimum sizes were larger than the critical size such as 100 molecules per cluster estimated by the theoretical calculation.

${ }^{1}$ M. A. Duncan and D. H. Rouvray, Sci. Am. 110 (Dec. 1989).

${ }^{2}$ M. L. Cohen and W. Knight, Phys. Today 42 (Dec. 1990).

${ }^{3}$ J. P. Devlin, J. Sadlej, and V. Buch, J. Phys. Chem. A 105, 974 (2001).

${ }^{4}$ D. M. Upadhyay, M. K. Shukla, and P. C. Mishra, Int. J. Quantum Chem. 81, 90 (2001).

${ }^{5}$ M. Ziskind, C. Mihesan, N. Lebrun, B. Chazallon, C. Focsa, and J. L. Destombes, Appl. Phys. A: Mater. Sci. Process. 79, 991 (2004).

${ }^{6}$ G. H. Takaoka, H. Noguchi, T. Yamamoto, and T. Seki, Jpn. J. Appl. Phys., Part 2 42, L1032 (2003).

${ }^{7}$ G. H. Takaoka, H. Noguchi, K. Nakayama, and M. Kawashita, e-J. Surf. Sci. Nanotechnol. 4, 473 (2006).

${ }^{8}$ G. H. Takaoka, K. Nakayama, D. Takeda, and M. Kawashita, Proceeding of the 16th International Conference on Ion Implantation Technology (IIT06) (AIP, New York, 2006), p. 190.

${ }^{9}$ P. Poulsen and D. R. Miller, Rarefied Gas Dynamics edited by J. L. Potter (AIAA, New York, 1977). Vol. 2, p. 899. 